\title{
THE HAFFKINE INSTITUTE
}

$\mathrm{T}$ HE annual report of the Haffkine Institute for 1959, which refers to the continued difficulty of recruiting qualified staff, includes lists of grants and research fellowships and scholarships as well as of papers published during the year*. Research activities in the Department of Antitoxins and Sera included. the antigenic analysis of cholera vaccine and the use of submerged or shaken cultures for growing diphtheria toxin and pertussis vaccine. In the Department of Chemotherapy, the synthesis of compounds of possible anti-tubercular activity continued, including heterocyclic derivatives of thiourea and thiocarbanilide. Compounds with the $p$-nitrophenyl and/or dichloroacetyl groups attached to the pyrimidine, thiazole and pyrazole ring were also synthesized and tested in vitro against Salmonella typhosa, while other pyrazole and pyrazolene derivatives have been synthesized for examination as anti-convulsants. Two 1 : 3-disubstituted sulphonyl ureas have shown promise as antidiabetics.

The Department of Clinical Pathology and Diagnostic Reagents has finally identified $E$. coli from urine by sero type (preliminary) of ' $O$ ' antigen determination and is pursuing its investigations on the antigenic structure of the typhoid-paratyphoid group under partial anaerobiosis for possible application in preparing improved agglutinable suspensions. Besides epidemiological studies of enteric infection, it has continued its antigenic work on cholera vaccine, its investigations on otomycoses and, in collaboration

* Government of Bombay. Annual Report of the Haffkine Inment Printing and 195 . P . 11 74 . (Bombay: Director, GovernCommissioner for India, 1960.) Rs. $0.60 ; 1 s$. with the Entomology Department and the Director's Laboratory, on the alimentary canal of the different rat-fleas with reference to the growth-promoting factors of some protozoa and bacteria. The Department of Entomology completed its studies on Drosophila muris, and started work on the morpho. logical structure of the salivary glands of different non-poisonous snakes in comparison with the parotid glands of the poisonous snakes. Besides work on cholera autotoxin, the Department of Pharmacology continued its work on indigenous drugs, and has developed a new colorimetric method for determining 5-chloro-7-iodo- and 5:7-diiodo-8-hydroxyquinoline by coupling with $p$-nitroaniline-2-sulphonic acid, and modified methods for determining m-aminophenyl in $p$-aminosalicylic acid have been examined as well as methods for determining 'Novalgin','Avil' and thymol.

In the Department of Virus Diseases the immunogenicity of viral vaccines was investigated with the view of standardizing testing procedures and work on the adaptation of locally isolated rabies street virus strains to embryonated eggs. The Biochemistry Section reports on preliminary methods of preserving tissues from cadavers, the screening of vegetable extracts for blood-group activity and the preparation of anti- $A$ and anti- $B$ serum, while the Department of Nutrition continued its nutritional evaluation of foodstuffs commonly consumed in Bombay State, and its investigations into the nutritive value of some leafy vegetables and their supplementary effect, as well as its work on fermented foods and on the effect of commercial sterilization on the nutritive value of milk.

\section{HOSPITAL IN-PATIENTS IN ENGLAND AND WALES}

\begin{abstract}
THE hospital in-patient inquiry for the years 1956-57 was organized jointly by the Ministry of Health and the General Register Office to provide statistics which will assist in the administration of the hospital services and contribute towards the knowledge of illness (morbidity) in the community*.

Information for the inquiry is supplied by hospitals, where the form is completed for one patient in every ten discharged, giving a confidential summary of the case record. Hospitals of all types except psychiatric take part in the inquiry which, since 1958 , has covered virtually all hospitals in England and Wales. In 1956 and 1957, the years to which the present report relates, the inquiry covered 68.5 and 82.1 per cent respectively of all hospital discharges and deaths in England and Wrles.

Apart from the main reference tables the report contains, for 1957, a series of tables devoted to duration of stay. This is related directly to bedusage, and the figures should be of value for planning purposes.
\end{abstract}

* Ministry of Health; and General Register Office. Report on Hospital In-Patient Enquiry for the two years 1956-195\%. Pp. vil + 335. (London: H.M.S.O., 1961.) 188. net.
Other features of the report are: a discussion of the statistical and other problems which arise in the interpretation of hospital morbidity data; some analyses according to the type of hospital in which patients were treated, and, for 'acute' hospitals, their size; a table showing age distribution for the 800 categories of the Detailed List of the International Statistical Classification of Diseases, and tables for six of the fully represented regions showing the local administrative area in which patients resided and the hospital group in which they were treated. These help to determine the 'catchment areas' of hospital groups.

Altogether, there were 3,485,000 discharges and deaths in 1957 in hospitals during the inquiry. The average duration of each spell of hospital care was slightly shorter than in 1955 or 1956 , being 22 days for males compared with 24 days in each of the two preceding years and 20 days for females compared with 22 ; in half the cases the duration of stay was less than $10 \frac{1}{2}$ days.

During 1957 maternity cases stayed an average of 10 days after delivery compared with 11 days in 1955. The proportion of mothers discharged within 7 days of delivery increased from 7 per cent in 1955 to 13 per cent in 1957. 51 per cent of mothers stayed 
8-10 days after delivery in 1957 and 36 per cent 11 days or more.

Children, aged 5-9 years, had the shortest average stay in hospital (11 days) and patients, aged 75 and over, the longest (45 days for men and 63 for women). Though this is mainly due to more chronic illness in older people, small children and older people stayed longer than older children and young adults for treatment of the same condition.

Of the patients aged 65 and more, women usually stayed longer than men; for circulatory diseases the average stay for men was 53 days and for women 72 , and for injuries other than head injuries 29 days for men and 41 days for women.

In 'acute' non-teaching hospitals the average length of stay was shortest in medium-sized hospitals. The same was true at all ages and for both sexes, and for many specific diagnoses.
From data on the duration of stay, the number of hospital beds required by different groups of patients can be calculated. The number of beds required per 10,000 population increases greatly with the patient's age, ranging from 20.8 at ages $15-24$ to 188.88 at ages 75 and more for men, and from 18.7 at ages 5-14 to 187.3 at ages 75 and more for women.

Of the 40.8 beds per 10,000 population used for male cases, 13 per cent were taken up by cases of respiratory tuberculosis ; 7 per cent were occupied in treatment of malignant neoplasms, 13 per cent for cardiovascular diseases, 10 per cent for respiratory diseases and 11 per cent for digestive diseases. For females, 48.9 beds per 10,000 population were in use; 7 per cent of them for respiratory tuberculosis, 6 per cent for malignant neoplasms, 8 per cent for digestive diseases and 15 per cent for maternity cases.

\section{RESPONSE TO HOT ENVIRONMENTS}

\begin{abstract}
A REPORT* prepared by the Medical Research Council owes its origin to the concern felt by the Board of Admiralty regarding the thermal conditions to which men were exposed during the Second World War when serving in His Majesty's ships in tropical waters. This lod, in 1944, to the appointment of a committee to inquire into and advise on the problems in this field. It was recognized from the outset that investigations would eventually have to be made in a tropical climate, and, in 1948, the Royal Naval Tropical Research Unit was established in Singapore under the joint auspices of the Admiralty and the Medical Research Council.
\end{abstract}

Before the establishment of the unit at Singapore, however, investigations were initiated in London at the National Hospital for Nervous Diseases. In the experiments carried out in London, the physiological effect on men wearing different types of clothing of exposure to high air temperatures was examined at different humidities and air speeds, both when the men were resting and when they were working. On the results of these experiments, for which the subjects had been artificially 'maximally acclimatized' by previous exposure to hot conditions in the laboratory, it was possible to base tentative recommendations concerning the conditions of warmth that were acceptable in ships at sea in tropical waters. The results also seemed to indicate that the "effective temperature scale', which had been accepted for use as a measure of the severity of environment in ships, was misleading in certain respects. This led Dr. B. McArdle to devise a new index, the 'predicted four-hour sweat-rate' (P4SR), for the assessment of hot environments.

With the establishment of the unit at Singapore similar investigations were carried out. These confirmed largely the previous findings in London and, though they did not entirely support the criticisms of the 'effective temperature scale', they did demonstrate that the 'predicted four-hour sweat-rate' was

* Privy Council. Medical Research Council Report Series, No. 298. Physiological Responses to Hot Environments. Compiled by $\dot{\mathbf{R}} . \mathbf{K}$. Macpherson. (An account of work done in Singapore, 1948-1953, at the Royal Naval Tropical Research Unit, with an appendix on preliminary work done at the National Hospital for Nervous Diseases,
London.) $P p$. $x v+323$. (London: H.M. Stationery Office, 1960.) 35s. net. an accurate and convenient measure of the severity of indoor hot-environments. The work at Singapore was, however, extended to compare the relative tolerance to heat of men who had been artificially 'maximally acclimatized' in the laboratory, with those who had been 'naturally acclimatized' by residence in a tropical climate. Investigations were also made of the stress imposed by radiant heat of long wavelength and by different rates of energy expenditure. Work carried out to determine the upper limits of warmth to be permitted in ships was extended to determine the upper limits that could be physically tolerated and the position of the 'comfort' zone. In addition, a number of physiological problems associated with these main lines of research was examined.

Now that the results of all the investigations in London and in Singapore have been brought together, it is clear that considerable additions to knowledge have been achieved. Much information has been obtained on the relative effects of various levels of air temperature, humidity, air speed, and radiant heat, and about the limits of heat which people can tolerate.

Answers have been provided to some of the practical problems of the Navy ; the critical examination of a widely used scale of environmental heat-stress and the production and testing of a new index have improved and added to the techniques available for evaluating the severity of different climatic environments. As well as achieving these practical ends, this work has extended knowledge of the physiological mechanisms involved in the process of adaptation to hot environments and has indicated the direction for future research.

The report has been compiled by Dr. R. K. Macpherson with the assistanc $\rightarrow$ of an editorial board composed of Dr. T. Bedford, Dr. O. G. Edholm, Surgeon Captain F. N. Ellis, and Dr. N. H. Mackworth. It brings together much patient and arduous work by a number of service-mon and civilians, and it is a fine example of how long-term investigations should be planned, organized and controlled. Many of its findings should be of interest to industrial organizations where hot processes are involved; managements in the new Indian steelworks should find much of value. 